\title{
Management of Metastatic Castration-Resistant Prostate Cancer
}

\author{
Presented by Sandy Srinivas, MD
}

\begin{abstract}
Notable developments in the management of metastatic castration-resistant prostate cancer (mCRPC) include newer, more sophisticated imaging methods based on prostate-specific membrane antigen (PSMA) PET and development of radionuclide ligands for use with this modality. In the therapeutic area, PSMA PET-guided therapeutics are under study, and PARP inhibitors are being used to treat patients with gene alterations directly or indirectly involved with the homologous recombination repair pathway. Cabazitaxel has emerged as an effective third-line option for patients treated with prior novel hormonal agents and/or prior docetaxel. As investigators learn more about sequencing therapies for $\mathrm{mCRPC}$, previous exposure is an important consideration for choice of treatment at disease progression.

J Natl Compr Canc Netw 2021;19(5.5):614-616
\end{abstract} doi: 10.6004/jnccn.2021.5003

Advances in the management of metastatic castrationresistant prostate cancer (mCRPC) include more sophisticated and sensitive imaging modalities that improve cancer detection and staging compared with conventional imaging methods. These methods can lead to earlier intervention when needed, stated Sandy Srinivas, MD, Professor of Medicine, Stanford Cancer Institute, at the NCCN 2021 Virtual Annual Conference. Furthermore, the newest imaging modalities use PET tracers to improve on sensitivity, and some of these tracers are showing promise when used as part of treatment.

PARP inhibitors represent another therapeutic advance and are currently being used to treat patients with genomic alterations in $H R R$ genes, including $B R C A$ somatic and germline alterations.

As the armamentarium for the treatment of mCRPC continues to expand, researchers are learning more about the optimal sequencing of available therapeutic options. "mCPRC represents the most advanced stage of prostate cancer, and patients will receive up to 4 or more lines of therapy at disease progression. The goal of treatment is to deliver effective, life-prolonging therapy to these patients, balancing longevity with quality of life," said Dr. Srinivas.

\section{Imaging Techniques}

Imaging is one of the cornerstones for the management of prostate cancer and is used to detect metastases, determine the number and location of metastases if present, and define tumor burden. A currently unmet need is a method that can evaluate response to therapy in bone disease, Dr. Srinivas explained. Conventional imaging techniques, such as bone scans, MRI, and CT, have shortcomings that appear to be overcome by more modern PET techniques.

"Bone scans are not specific to prostate cancer and don't evaluate response to therapy. CT scans mostly visualize lymph node disease in the chest, retroperitoneum, or pelvis. MRI scans have tried to improve upon the detection of lymph nodes, but there is an unmet need for more functional imaging in prostate cancer," Dr. Srinivas stated.

Several PET scan molecules are currently available, 4 of which are FDA-approved and include 18F-fluciclovine and ${ }^{68} \mathrm{Ga}-\mathrm{PSMA}-11$ (Table 1 ). However, these have limitations and are not widely available. ${ }^{18} \mathrm{~F}$-DCFPyL is investigational and may ultimately become useful in prostate cancer. Prostate-specific membrane antigen (PSMA) PET is the most promising of the newer techniques. PSMA is overexpressed almost universally by mCRPC cells and represents an ideal target for molecular imaging when labeled with radionuclides such as ${ }^{68} \mathrm{Ga}$ or ${ }^{18} \mathrm{~F}$-. Numerous studies have shown that PSMA PET is superior to conventional imaging for the detection of metastases in patients with prostate cancer. ${ }^{1-3}$

"PSMA PET will change the way we perform imaging in prostate cancer," Dr. Srinivas predicted. "We are now capitalizing on the extracellular domain [by targeting PSMA] with PSMA ligands, and this is opening up the field," she said. "Most of these radionuclides will be similar, and a PSMA PET should perform equally well with each of these compounds."

The OSPREY study evaluated the experimental radionuclide ${ }^{18} \mathrm{~F}$-DCFPyL PET in 385 men with locally recurrent mCRPC and compared findings with biopsies. ${ }^{4}$ Cohort A included 268 men with high-risk prostate 


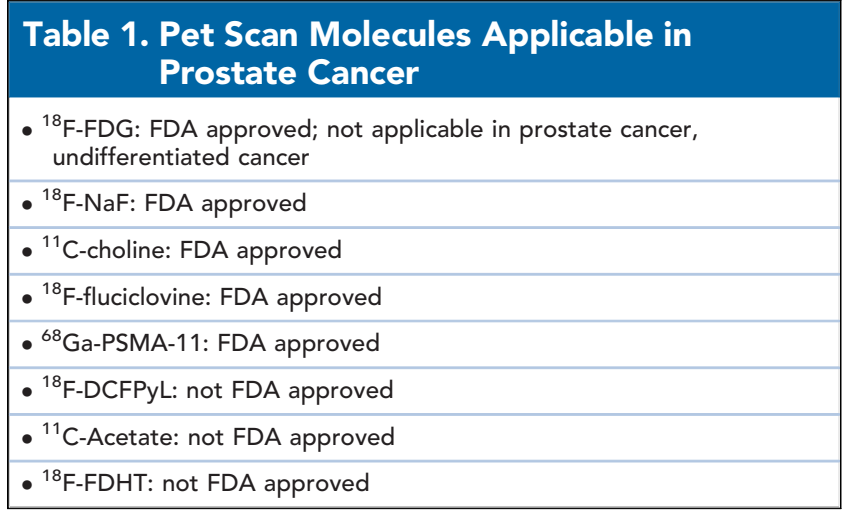

cancer treated with ${ }^{18} \mathrm{~F}$-DCFPyL (safety set); of these, 252 underwent PET/CT imaging and had evaluable surgical pathology (evaluable set). Cohort B comprised 117 men with recurrent or mCRPC treated with ${ }^{18} \mathrm{~F}$-DCFPyL (safety set), and of these, 93 had PET/CT imaging and evaluable pathology from image-guided biopsy (evaluable set). In cohort B, ${ }^{18} \mathrm{~F}$-DCFPyL had a sensitivity of $95 \%$ to $100 \%$ for detection of bone, lymph node, and visceral/soft tissue metastases related to histology; the positive predictive value of ${ }^{18} \mathrm{~F}$-DCFPyL was $\geq 80 \%$.

In a separate study of 200 men with high-risk nonmetastatic prostate cancer, ${ }^{5}$ the investigators determined that PSMA PET detected metastasis in 196 patients despite their being staged as nonmetastatic; $55 \%$ of these men with PSMA PET-detected metastasis had negative findings on conventional imaging.

A framework for use of next-generation imaging (NGI) was proposed by RADAR3 investigators. ${ }^{6}$ In newly diagnosed patients, NGI can be considered when conventional imaging is equivocal or negative with continued high suspicion of metastatic disease. For biochemically recurrent prostate cancer, NGI should be considered for a prostate-specific antigen (PSA) level $\geq 0.5 \mathrm{ng} / \mathrm{mL}$. For non-mCRPC, NGI should be considered only in the setting of PSA doubling time $<6$ months when first-line metastatic therapy would be appropriate. For mCRPC, conventional imaging should be used and NGI should be considered only if conventional scans are negative and there is a high suspicion of disease progression due to PSA doubling time, change in symptoms, and change in performance status.

"Imaging is highly integrated in decision-making for prostate cancer. Agents such as ${ }^{18} \mathrm{~F}$-fluciclovine, ${ }^{68} \mathrm{Ga}-$ PSMA-11, ${ }^{18} \mathrm{~F}$-DCFPyL, and ${ }^{11} \mathrm{C}$-choline show promise in detecting the presence of metastasis versus no metastasis [and] high-volume versus low-volume disease, guidance of metastases-directed therapy, and treatment of oligometastatic disease," Dr. Srinivas said. "Theranostics [ie, combining drugs for diagnosis and treatment of medical conditions] is coming in the near future."

\section{PARP Inhibitors}

One of the more recent advances in treating MCRPC is the use of PARP inhibitors in patients with genomic alterations in the homologous recombination repair (HRR) pathway. The phase III PROfound study compared the PARP inhibitor olaparib versus physician's choice in 387 men with progressive mCRPC. ${ }^{7,8}$ Patients enrolled in this study had experienced prior disease progression on next-generation hormonal therapy (either enzalutamide or abiraterone); additionally, two-thirds also had prior chemotherapy. Cohort A included 245 men with BRCA1, BRCA2, or ATM alterations, and cohort B included 142 men with any of 12 other prespecified alterations in the HRR pathway. Both cohorts were randomized 2:1 to treatment with olaparib or physician's choice of enzalutamide or abiraterone. Crossover to olaparib was allowed on progression of physician's choice of therapy (either enzalutamide or abiraterone, dependent on previous exposure), and approximately $66 \%$ of patients in both cohorts crossed over.

Radiographic progression-free survival was significantly improved in patients assigned to olaparib in cohort A (7.4 vs 3.6 months for physician's choice; $P<.001$ ) and was also significantly improved in the overall population treated with olaparib versus physician's choice, despite a high rate of crossover to olaparib. The secondary endpoint of pain progression also favored olaparib in both cohorts A and B. ${ }^{8}$

Overall survival was significantly improved in patients assigned to olaparib in cohort A (19.1 vs 14.7 months for physician's choice; $P=.02$ ). In cohort $\mathrm{B}$, median overall survival was 14.1 versus 11.5 months, respectively, a difference that was not statistically significant. Overall, in both cohorts, median overall survival was 17.3 versus 14.0 months. These results held up in a sensitivity analysis adjusted for the fact that two-thirds of patients originally assigned to physician's choice crossed over to olaparib on disease progression. ${ }^{7}$

"For the entire cohort of the PROfound study, most of the benefit of olaparib was observed in BRCA-positive and ATM-positive tumors. The number of patients with other genetic alterations are too small to draw conclusions [on the benefit of olaparib]," Dr. Srinivas said.

TRITON2 was a phase II, single-arm study that evaluated the PARP inhibitor rucaparib in patients with deleterious or somatic mutations in HRR genes who progressed on prior therapy with an androgen receptor-directed therapy and one prior taxane-based chemotherapy for mCRPC. ${ }^{9}$ Overall, independently assessed response rates of $44 \%$ led to the accelerated FDA approval of rucaparib as treatment in patients with mCRPC and BRCA mutations previously treated with a next-generation hormonal agent and taxane.

PARP inhibitors continue to be an active area of investigation in prostate cancer, with at least 5 phase III 
Table 2. Ongoing Trials of PARP Inhibitors in Prostate Cancer

\begin{tabular}{|c|c|c|c|}
\hline Drug & Phase & Description & Endpoint \\
\hline Rucaparib & III & Rucaparib vs patient/physician choice (TRITON3a) & PFS \\
\hline Olaparib & III & Olaparib + pembrolizumab vs Abi or Enza (KEYLINK $\left.{ }^{b}\right)$ & $\mathrm{PFS} / \mathrm{OS}$ \\
\hline Niraparib & III & Niraparib + Abi vs Abi (cohort 1 with HRD enrichment only) (MAGNITUDE ${ }^{c}$ ) & PFS \\
\hline Olaparib & III & Olaparib + Abi vs placebo + Abi $\left(\right.$ PROpel $\left.{ }^{d}\right)$ & PFS \\
\hline Talazoparib & III & Talazoparib + Enza vs placebo + Enza (TALAPRO-2 ${ }^{\mathrm{e}}$ ); pre-chemotherapy & PFS \\
\hline
\end{tabular}

Abbreviations: Abi, abiraterone acetate; Enza, enzalutamide; HRD, homologous recombination repair deficiency; OS, overall survival; PFS, progression-free survival.

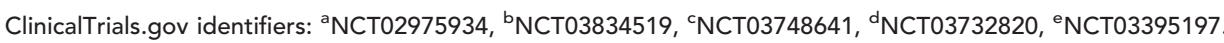

trials that are currently ongoing or completed (Table 2). Some of these trials are combination studies.

The latest NCCN Clinical Practice Guidelines in Oncology (NCCN Guidelines) for Prostate Cancer (Version 2.2021$)^{10}$ incorporate both olaparib and rucaparib as options for patients who have experienced disease progression on prior novel hormonal therapy and have not had docetaxel, and for those who have had prior novel hormonal therapy and prior docetaxel. Olaparib is recommended for patients with HRR alterations, and rucaparib is restricted to patients with $B R C A$ mutations.

More recently, the CARD trial demonstrated that cabazitaxel was an effective third-line therapy in patients who had experienced disease progression on novel androgen receptor-targeted therapy with or without prior docetaxel. ${ }^{11}$ CARD was a randomized phase III trial comparing cabazitaxel versus enzalutamide or abiraterone in 255 men with MCRPC whose disease had progressed on prior docetaxel and either abiraterone or enzalutamide. Median progression-free survival was 4.4 months with cabazitaxel and 2.7 months with an androgen signalingtargeted inhibitor $(P<.001)$. A PSA response occurred in $35.7 \%$ and $13.5 \%$ of patients, respectively $(P<.001)$, and tumor response was noted in $36.5 \%$ and $11.5 \%$, respectively ( $P=.004$ ). Grade $\geq 3$ adverse events occurred in $56.3 \%$ of patients receiving cabazitaxel and in $52.4 \%$ of those receiving an androgen signaling-targeted inhibitor. No new safety signals were observed.

"The CARD trial reinforced what we have learnedthat interspersing chemotherapy is the best choice for patients coming off of novel hormonal therapy," Dr. Srinivas stated. The most recent NCCN Guidelines ${ }^{10}$ incorporate use of cabazitaxel or pembrolizumab as useful in certain circumstances (ie, patients with microsatellite instability-high tumors) for patients treated with prior novel hormonal agents with or without prior docetaxel. The choice of agent is currently based on prior exposure, she explained. For patients exposed to prior docetaxel, novel hormonal therapy would be a good option. Cabazitaxel would be a good option for those exposed to both prior novel hormonal agents and prior docetaxel. Patients with alterations in HRR genes would be candidates for a PARP inhibitor.

Disclosures: Dr. Srinivas has disclosed receiving grant/research suppor from Endocyte, Exelixis Inc., and Bayer HealthCare; and serving as a scientific advisor for Bayer HealthCare.

Correspondence: Sandy Srinivas, MD, Stanford Cancer Institute, 875 Blake Wilbur Drive, Stanford, CA 94305. Email: sandysri@stanford.edu

\section{References}

1. Davis MI, Bennett MJ, Thomas LM, Bjorkman PJ. Crystal structure of prostate-specific membrane antigen, a tumor marker and peptidase. Proc Natl Acad Sci U S A 2005;102:5981-5986.

2. Eder $M$, Schäfer $M$, Bauder-Wüst $U$ et al. 68Ga-complex lipophilicity and the targeting property of a urea-based PSMA inhibitor for PET imaging. Bioconjug Chem 2012;23:688-697.

3. Barinka $C$, Byun $Y$, Dusich $C L$, et al. Interactions between human glutamate carboxypeptidase II and urea-based inhibitors: structural characterization. J Med Chem 2008;51:7737-7743.

4. Pienta KS, Gorin MA, Rowe SP. A phase $2 / 3$ prospective multicenter study of the diagnostic accuracy of prostate-specific membrane antigen PET/CT with 18 F-DCFPyL in prostate cancer patients (OSPREY) [published online February 26, 2021]. J Urol, doi: 10.1097/JU. 0000000000001698

5. Fendler W, Weber M, Iravani A, et al. Prostate-specific membrane antigen ligand positron emission tomography in met with non-metastatic castration-resistant prostate cancer. Clin Cancer Res 2019;25:7448-7434.
6. Crawford ED, Schellhammer PF, McLeod DG, et al. Androgen receptor targeted treatments of prostate cancer: 35 years of progress with antiandrogens. J Urol 2018;200:956-966.

7. Hussain M, Mateo J, Fizazi K, et al. Survival with olaparib in metastatic castration-resistant prostate cancer. N Engl J Med 2020;383:2345-2357.

8. deBono J, Mateo J, Fizazi K, et al. Olaparib in metastatic castration-resistant prostate cancer. N Engl J Med 2020;382:2091-2102.

9. Abida A, Patnaik A, Campbell D, et al. Rucaparib in men with metastatic castration-resistant prostate cancer harboring a BRCA1 or BRCA2 alteration. J Clin Oncol 2020;38:3763-3772.

10. Schaeffer E, Srinivas S, Antonarakis ES, et al. NCCN Clinical Practice Guidelines in Oncology: Prostate Cancer. Version 2.2021. Accessed April 1, 2021. To view the most recent version, visit NCCN.org

11. DeWit R, de Bono J, Sternberg CN, et al. Cabazitaxel versus abiraterone or enzalutamide in prostate cancer. $\mathrm{N}$ Engl J Med 2019;381:2506-2518. 